\title{
The epipelic algae of fishponds of Central and Northern Moravia (The Czech Republic)
}

\author{
Monika LYSÁKOVÁ, Miloslav KITNER \& Aloisie POULíČKOVÁ \\ Department of Botany, Faculty of Science, Šlechtitelì 11, CZ-78371 Olomouc-Holice, Czech Republic.
}

\begin{abstract}
Epipelic assemblages have not been studied in the Czech Republic yet. The sampling method following Round (1953) was used for the fist time in the Czech Republic. The epipelic assemblages were dominated by diatoms, particularly in mesotrophic and meso-eutrophic fishponds, eutrophic fishponds were dominated by green algae and cyanobacteria. A total of 130 diatom species were found in 16 fishponds, their list with trophic preferences is presented. Seven morphospecies were distinquished within Sellaphora pupula species complex, their clonal cultures will be studied later by molecular methods.
\end{abstract}

Key words: epipelon, diatoms, ponds, diversity, Sellaphora

\section{Introduction}

Fishponds represent the most common type of stagnant water biotopes in the Czech Republic. Their investigation has had a long tradition, but has mainly focused on phytoplankton (KOŘíNEK et al. 1987) and its primary production, especially in large fish-producing systems in the southern part of the Czech Republic (Třeboň, Lednice). Littoral periphytic algae have been studied only occasionally (KOMÁRKOVÁ \& MARVAN 1987, KITNER \& POULÍČKOVÁ 2003, POULÍČKOVÁ et al. 2003). Epipelic assemblages have not been studied yet. An easy method of their sampling (RouND 1953) was used for the first time in this study.

The epipelon is an extremely widespread community occurring in all waters in regions where sediments accumulate and on to which light penetrates (lakes, ponds, pools, slowly flowing rivers). The species composition of the epipelon varies widely between habitats, though it tends to be fairly constant for any one type of water. Thanks to these features, it can be successfully used for stagnant water biomonitoring, which still has no standard method in the EU in contrast to rivers (POULÍČKOVÁ et al. 2004).

The use of diatoms for monitoring ecological conditions has had quite a long tradition also in the Czech Republic and is planned to continue, especially following the Water Framework Directive. The success of diatom monitoring depends critically on 1) taxonomic precision (are diatom species equivalent to species elsewhere, or are they equivalent to genera of angiosperms?, etc.), 2) biogeography of diatoms - are all diatoms cosmopolitan as claimed e.g. by FINLAY et al. (2002) or not (MANN 1999)? and 3) consistent use of names. All of these are compromised by lack of understanding of nature of species.

Traditionally, diatom taxonomy depends on cell wall characteristics, but there has been no consensus on where species boundaries should be drawn. So far most studies indicate that diatom species are often heterogenous complexes, containing several to many semicryptic or cryptic species. Although epipelic species complexes, particularly Sellaphora pupula agg., have been studied for a long time in British lakes and ponds, there is still no information about ecological preferences of diatom epipelic species, and only a little is known about the diversity and distribution of these species complexes in the rest of Europe.

The presented paper focuses on the species composition of the studied epipelic assemblages and their potential use in the assessment of trophic status of fishponds.

\section{Methods}

Altogether 16 localities (Central Moravia, The Czech Republic) were investigated at the end of the season in September 2005. Phytoplankton, littoral attached algae and ecological variables had been studied 


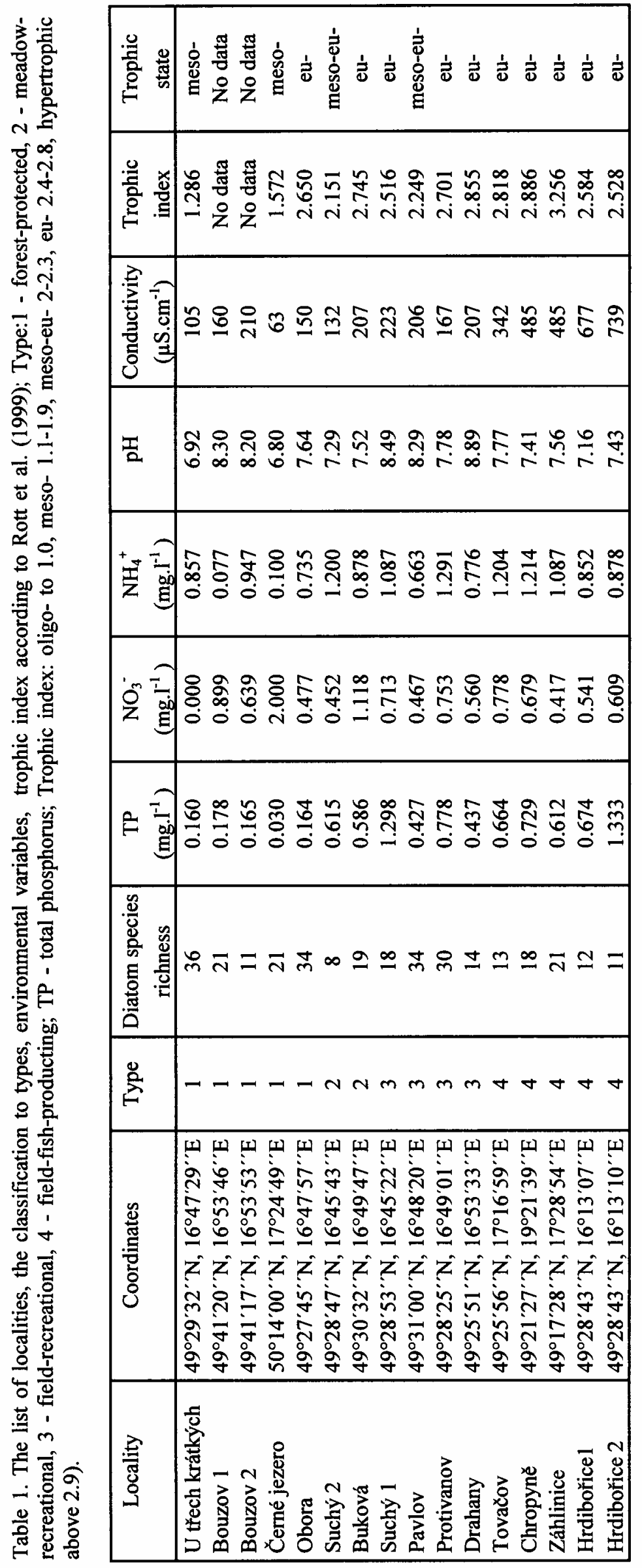


at the same sites previously (KITNER \& POULÍČKOVÁ 2003). The altitude of investigated sites ranges from 490 to $660 \mathrm{~m}$ a.s.l., their area ranges from 0,068 ha to 5.52 ha, their maximal depth is $75-280 \mathrm{~cm}$ and shading $5-85 \%$. Tab. 1 summarizes the trophic characteristics of the sites.

Surface sediments and overlying water were collected using a glass tube, as described by Round (1953), transported to the laboratory in polyethylene bottles, poured out into plastic boxes, and allowed to stand in the dark for at least $5 \mathrm{~h}$. Then the supernatant was removed by suction and the mud covered with lens tissue. Under continuous low-level illumination (circa $5 \mu$ mol photons. $\mathrm{m}^{-2} \cdot \mathrm{s}^{-1}$ ), epipelic algae moved up through the lens tissue and became attached to cover slips placed on top. The cover slips were collected from the mud after 12 and $24 \mathrm{~h}$ of exposition. The algae were counted on the identical area of the cover slip $\left(22 \mathrm{~mm}^{2}\right)$.

Cleaned valves were prepared with boiling $30 \%$ hydrogen peroxide, followed by washing with distilled water. After washing, the valves were mounted in Naphrax.

\section{Results and discussion}

The epipelic assemblages were represented by Bacillariophyceae, Chlorophyta, Cyanophyta,
Euglenophyta, Dinophyta and Cryptophyta (Tab. 2), most of them being dominated by diatoms (Tab. 3). The representation of epipelic diatom species on cover slips were similar when collected after 12 or $24 \mathrm{~h}$, with the exception of large diatoms (Amphora ovalis, Pinnularia gibba) which colonize a trap after $24 \mathrm{~h}$. Although the species representation seems to be similar to that studied in England (PoulíčKOVÁ et al. 2007), we cannot be sure that morphospecies distinquised within the species complexes (Sellaphora pupula agg. and others) are genetically identical. Our research will continue in two directions: (1) Diversity and distribution of epipelic diatom species and their ecological preferences, particularly Navicula cryptocephala agg., Sellaphora pupula agg., Amphora copulata, Pinnularia gibba agg., Cymatopleura solea agg. (2) Determination of the relationship between morphological and cytological variation, mating compatibility and genetic population diversity in selected epipelic pennate diatoms.

Table 2. Representation of cyanobacteria and algae in the fishponds under investigation.

\begin{tabular}{|c|c|c|c|c|c|c|c|c|}
\hline \multirow[t]{2}{*}{ Algal groups } & \multicolumn{8}{|c|}{ Locality } \\
\hline & Buková & Pavlov & Suchá 1 & Suchá 2 & Protivanov & Obora & $\begin{array}{c}\text { Hrdibořice } \\
1\end{array}$ & $\begin{array}{c}\text { Hrdibořice } \\
2\end{array}$ \\
\hline Chlorophyta & $19 \%$ & $9 \%$ & $24 \%$ & $30 \%$ & $22 \%$ & $15 \%$ & $33 \%$ & $34 \%$ \\
\hline Cyanophyta & $10 \%$ & $4 \%$ & $24 \%$ & $30 \%$ & $19 \%$ & $19 \%$ & $22 \%$ & $21 \%$ \\
\hline Euglenophyta & $14 \%$ & $13 \%$ & $10 \%$ & $10 \%$ & $7 \%$ & $4 \%$ & $8 \%$ & $11 \%$ \\
\hline Cryptophyta & $5 \%$ & $4 \%$ & $0 \%$ & $0 \%$ & $4 \%$ & $0 \%$ & $0 \%$ & $0 \%$ \\
\hline Dinophyta & $10 \%$ & $0 \%$ & $0 \%$ & $0 \%$ & $0 \%$ & $0 \%$ & $4 \%$ & $0 \%$ \\
\hline \multirow[t]{2}{*}{ Bacillariophyceae } & $42 \%$ & $70 \%$ & $42 \%$ & $30 \%$ & $48 \%$ & $62 \%$ & $33 \%$ & $34 \%$ \\
\hline & Záhlinice & Tovačov & Chropyně & Bouzov 1 & Bouzov 2 & Drahany & $\begin{array}{l}\text { Černé } \\
\text { jezero }\end{array}$ & $\begin{array}{l}\text { U třech } \\
\text { krátkých }\end{array}$ \\
\hline Chlorophyta & $29 \%$ & $25 \%$ & $25 \%$ & $37 \%$ & $50 \%$ & $35 \%$ & $21 \%$ & $12 \%$ \\
\hline Cyanophyta & $8 \%$ & $21 \%$ & $30 \%$ & $22 \%$ & $11 \%$ & $17 \%$ & $21 \%$ & $15 \%$ \\
\hline Euglenophyta & $13 \%$ & $12 \%$ & $10 \%$ & $11 \%$ & $17 \%$ & $13 \%$ & $13 \%$ & $12 \%$ \\
\hline Cryptophyta & $4 \%$ & $4 \%$ & $5 \%$ & $0 \%$ & $0 \%$ & $4 \%$ & $4 \%$ & $0 \%$ \\
\hline Dinophyta & $0 \%$ & $0 \%$ & $0 \%$ & $0 \%$ & $0 \%$ & $0 \%$ & $0 \%$ & $4 \%$ \\
\hline Bacillariophyceae & $46 \%$ & $38 \%$ & $30 \%$ & $30 \%$ & $22 \%$ & $31 \%$ & $42 \%$ & $57 \%$ \\
\hline
\end{tabular}


Table 3. List of taxa (Bacillariophyceae) in 16 fishponds of Northern and Central Moravia. Locality: 1 U třech krátkých, 2 Bouzov 1, 3 Bouzov 2, 4 Černé jezero, 5 Obora, 6 Suchý 2, 7 Buková, 8 Suchý 1, 9 Pavlov, 10 Protivanov, 11 Drahany, 12 Tovačov, 13 Chropyně, 14 Záhlinice, 15 Hrdibořice 1, 16 Hrdibořice 2; Occurrence: 1 rare, 2 frequent, 3 common; trophic preferences: our data based on 16 fishponds. Nomenclature according to Krammer \& Lange-Bertalot 1986, 1988, 1991 except of Sellaphora sp. comp.

\begin{tabular}{|c|c|c|c|}
\hline Taxa & Locality & Occurrence & $\begin{array}{c}\text { Trophic } \\
\text { preferences }\end{array}$ \\
\hline $\begin{array}{l}\text { Achnanthes altaica (V.S. PORETZKY) } \\
\text { CLEVE - EULER }\end{array}$ & 15,16 & 1 & eutrof \\
\hline $\begin{array}{l}\text { Achnanthes helvetica (HUST.) LANGE - } \\
\text { BERT. }\end{array}$ & 8 & 1 & eutrof \\
\hline Achnanthes lanceolata (BRÉBI.) GRUNOW & $1,5,7$ & 1 & $\begin{array}{l}\text { meso- } \\
\text { eutrof }\end{array}$ \\
\hline Achnanthes minutisima KüTZ. & 1 & 1 & meso \\
\hline $\begin{array}{l}\text { Anomoeoneis sphaerophora (EHRENB.) } \\
\text { PFITZER }\end{array}$ & 13,16 & 1 & eutrof \\
\hline Anomoeoneis vitrea (GRUNOw) Ross & 1,12 & 1 & $\begin{array}{l}\text { meso- } \\
\text { eutrof }\end{array}$ \\
\hline Amphora ovalis KüTZ. & $1,9,10,11,12$ & 2 & $\begin{array}{l}\text { meso- } \\
\text { eutrof }\end{array}$ \\
\hline Asterionella formosa HASSAL & 5 & 1 & eutrof \\
\hline $\begin{array}{l}\text { Aulacoseira alpigena GRUNOW } \\
\text { (KRAMMER) }\end{array}$ & $10,12,15$ & 1 & eutrof \\
\hline $\begin{array}{l}\text { Aulacoseira granulata (EHRENB.) } \\
\text { SIMONSEN }\end{array}$ & $5,9,10,12,14,16$ & 2 & $\begin{array}{l}\text { meso- } \\
\text { eutrof }\end{array}$ \\
\hline Aulacoseira lirata (EHRENB.) Ross & 7 & 1 & eutrof \\
\hline Caloneis silicula (EHRENB.) CLEVE & 4,9 & 1 & $\begin{array}{l}\text { meso- } \\
\text { eutrof }\end{array}$ \\
\hline Cocconeis placentula EHRENB. & $1,6,9,10$ & 2 & $\begin{array}{l}\text { meso- } \\
\text { eutrof }\end{array}$ \\
\hline Cyclotella atomus Hust. & $\begin{array}{c}1,2,3,5,7,8,9,10,11,12,13 \\
14,15,16\end{array}$ & 3 & $\begin{array}{l}\text { meso- } \\
\text { eutrof }\end{array}$ \\
\hline Cyclotella distinguenda Hust. & 2,5 & 1 & eutrof \\
\hline Cyclotella dubia (Fricke) Round & 10 & 1 & eutrof \\
\hline Cyclotella meneghiniana KÜTZ. & $1,3,6,7,9,12,13,14$ & 2 & $\begin{array}{l}\text { meso- } \\
\text { eutrof }\end{array}$ \\
\hline Cyclotella pseudostelligera Hust. & $3,7,10,13$ & 1 & eutrof \\
\hline Cyclotella stelligera CLEVE et GRUNOW & 5 & 1 & eutrof \\
\hline Cymbella affinis KüTZ. & 14 & 1 & eutrof \\
\hline Cymbella amphicephala NäGELI & 1,5 & 1 & $\begin{array}{l}\text { meso- } \\
\text { eutrof }\end{array}$ \\
\hline Cymbella cistula (EHRENB.) KIRCHN. & 6 & 1 & $\begin{array}{l}\text { meso- } \\
\text { eutrof }\end{array}$ \\
\hline Cymbella descripta (Hust.) KRAMMER & 4 & 1 & meso \\
\hline Cymbella gracilis (EHRENB.) KüTZ. & $1,4,8$ & 1 & $\begin{array}{l}\text { meso- } \\
\text { eutrof }\end{array}$ \\
\hline Cymbella minuta HILSE & 9 & 1 & $\begin{array}{l}\text { meso- } \\
\text { eutrof }\end{array}$ \\
\hline Cymbella naviculiformis AVERSWALD & 4 & 1 & meso \\
\hline Cymbella silesiaca BLEISCH & $2,5,9,10,15$ & 2 & $\begin{array}{l}\text { meso- } \\
\text { eutrof }\end{array}$ \\
\hline Cymbella tumida (BRÉB.) HeUrCK & 2,10 & 1 & eutrof \\
\hline Epithemia sorex KÜTZ. & 16 & 1 & eutrof \\
\hline Eunotia bilunaris (EHRENB.) MILLS & 8 & 1 & eutrof \\
\hline Eunotia incisa W.GREG. & 1 & 1 & meso \\
\hline Eunotia exigua (BRÉB.) RABENH. & $1,4,8$ & 1 & $\begin{array}{l}\text { meso- } \\
\text { eutrof }\end{array}$ \\
\hline Eunotia minor (KÜTZ.) GRUNOW & 5 & 1 & eutrof \\
\hline Eunotia paludosa GRUNOW & 8 & 1 & eutrof \\
\hline
\end{tabular}




\begin{tabular}{|c|c|c|c|}
\hline Cont. Table 3 & & & \\
\hline Fragilaria bicapitata A. MAYER & 1,5 & 1 & $\begin{array}{l}\text { meso- } \\
\text { eutrof }\end{array}$ \\
\hline Fragilaria capucina DESM. & $1,9,10$ & 1 & $\begin{array}{l}\text { meso- } \\
\text { eutrof }\end{array}$ \\
\hline $\begin{array}{l}\text { Fragilaria construens (EHRENB.) } \\
\text { GRUNOW }\end{array}$ & 1,9 & 1 & $\begin{array}{l}\text { meso- } \\
\text { eutrof }\end{array}$ \\
\hline Fragilarie elliptica SснUм. & 8 & 1 & eutrof \\
\hline $\begin{array}{l}\text { Fragilaria parasitica (W.SмITH) } \\
\text { GRUNOW }\end{array}$ & 1,5 & 1 & $\begin{array}{l}\text { meso- } \\
\text { eutrof }\end{array}$ \\
\hline Fragilaria pinnata EHRENB. & $9,15,16$ & 1 & $\begin{array}{l}\text { meso- } \\
\text { eutrof }\end{array}$ \\
\hline Fragilaria virescens RALFS & 1 & 1 & meso \\
\hline Gomphonema acuminatum EHRENB. & $1,7,10,11$ & 2 & $\begin{array}{l}\text { meso- } \\
\text { eutrof }\end{array}$ \\
\hline Gomphonema affine KüTZ. & 10 & 1 & eutrof \\
\hline $\begin{array}{l}\text { Gomphonema angustatum (KÜTZ.) } \\
\text { GRUNOW }\end{array}$ & 1,2 & 1 & $\begin{array}{l}\text { meso- } \\
\text { eutrof }\end{array}$ \\
\hline Gomphonema gracile EHRENB. & $1,4,5,8,9,11$ & 2 & $\begin{array}{l}\text { meso- } \\
\text { eutrof }\end{array}$ \\
\hline $\begin{array}{l}\text { Gomphonema olivaceum (HORNEM.) } \\
\text { BRÉB. }\end{array}$ & 6 & 1 & $\begin{array}{l}\text { meso- } \\
\text { eutrof }\end{array}$ \\
\hline $\begin{array}{l}\text { Gomphonema parvulum (KÜTZ.) } \\
\text { GRUNOW }\end{array}$ & $5,7,8,10,11,12,14,15,16$ & 3 & eutrof \\
\hline Gomphonema truncatum EHRENB. & 1 & 1 & meso \\
\hline $\begin{array}{l}\text { Gyrosigma acuminatum (KÜTZ.) } \\
\text { RABENH. }\end{array}$ & 14 & 1 & eutrof \\
\hline Melosira varians AGARDH & 9 & 1 & $\begin{array}{l}\text { meso- } \\
\text { eutrof }\end{array}$ \\
\hline Navicula accomoda HUST. & 14 & 1 & eutrof \\
\hline Navicula angusta GRUNOW & 2 & 1 & eutrof \\
\hline Navicula atomus (KÜTZ.) GRUNOW & 2 & 1 & eutrof \\
\hline Navicula capitata EHRENB. & $2,4,5,9,10,13$ & 2 & $\begin{array}{l}\text { meso- } \\
\text { eutrof }\end{array}$ \\
\hline Navicula cincta (EHRENB.) RALFS & 13 & 1 & eutrof \\
\hline Navicula constans HuST. & 1,5 & 1 & $\begin{array}{l}\text { meso- } \\
\text { eutrof }\end{array}$ \\
\hline Navicula cryptocephala KüTz. & $1,2,4,5,7,8,9,10,11,13,15,16$ & 3 & $\begin{array}{l}\text { meso- } \\
\text { eutrof }\end{array}$ \\
\hline Navicula cuspidata (KÜTZ.) KüTZ. & 13,14 & 1 & eutrof \\
\hline Navicula elginensis (GREG.) RALFS & 4 & 1 & meso \\
\hline Navicula gallica (W.SMITH) LAGERST. & $2,3,9,10,15$ & 2 & $\begin{array}{l}\text { meso- } \\
\text { eutrof }\end{array}$ \\
\hline Navicula gregaria DONKIN & 3,10 & 1 & eutrof \\
\hline Navicula halophila (GRUNOW) CLEVE & 4,7 & 1 & $\begin{array}{l}\text { meso- } \\
\text { eutrof }\end{array}$ \\
\hline Navicula lenzii HuST. & 10 & 1 & eutrof \\
\hline Navicula menisculus SCHUM. & 2,14 & 1 & eutrof \\
\hline Navicula minima GRUNOW & $5,11,13$ & 1 & eutrof \\
\hline Navicula phyllepta KüTz. & 9,10 & 1 & eutrof \\
\hline Navicula radiosa KÜTZ. & 4 & 1 & meso \\
\hline Navicula rhynchocephala KÜTZ. & 5,9 & 1 & $\begin{array}{l}\text { meso- } \\
\text { eutrof }\end{array}$ \\
\hline Navicula seminulum GRUNOW & 7 & 1 & eutrof \\
\hline Navicula schoenfeldii Hust. & 14 & 1 & eutrof \\
\hline Navicula similis KRASSKE & 11 & 1 & eutrof \\
\hline Navicula species LANGE-BERT. & 12 & 1 & eutrof \\
\hline Navicula subtilissima CLEVE & 7 & 1 & eutrof \\
\hline Navicula trivialis LANGE-BERT. & $2,9,13$ & 1 & $\begin{array}{l}\text { meso- } \\
\text { eutrof }\end{array}$ \\
\hline Navicula viridula (KüTZ.) KüTZ. & 3 & 1 & eutrof \\
\hline
\end{tabular}




\begin{tabular}{|c|c|c|c|}
\hline Cont. Table 3 & & & \\
\hline Navicula veneta KüTZ. & 6,14 & 1 & $\begin{array}{l}\text { meso- } \\
\text { eutrof }\end{array}$ \\
\hline Neidium ampliatum (EHRENB.) & $1,4,5,8,9$ & 2 & meso- \\
\hline KRAMMER & & & eutrof \\
\hline Neidium productum (W. SMITH) CLEVE & 8,14 & 1 & eutrof \\
\hline Nitzschia acicularis W. SмIтH & $3,5,6,12,14,15,16$ & 2 & $\begin{array}{l}\text { meso- } \\
\text { eutrof }\end{array}$ \\
\hline $\begin{array}{l}\text { Nitzschia actinastroides (LEMMERM.) V. } \\
\text { GOOR }\end{array}$ & $3,7,10$ & 1 & eutrof \\
\hline Nitzschia agnita Hust. & 1 & 1 & meso \\
\hline Nitzschia calida GRUNOW & 13 & 1 & eutrof \\
\hline Nitzschia capitellata HusT. & 13,14 & 1 & eutrof \\
\hline Nitzschia fonticola GRUNOW & 14 & 1 & eutrof \\
\hline Nitzschia fruticosa HUST. & 11 & 1 & eutrof \\
\hline Nitzschia gracilis HANTZSCH & $4,6,10,14$ & 2 & $\begin{array}{l}\text { meso- } \\
\text { eutrof }\end{array}$ \\
\hline Nitzschia inconspicua GRUNOW & 3 & 1 & eutrof \\
\hline Nitzschia intermedia HANTZSCH & $1,2,7,9$ & 2 & $\begin{array}{l}\text { meso- } \\
\text { eutrof }\end{array}$ \\
\hline Nitzschia linearis (AGARDH) W. SMITH & 4 & 1 & meso \\
\hline Nitzschia palea (Kütz.) W. SмIтн & $1,2,4,5,7,9,11,12,13,16$ & 3 & $\begin{array}{l}\text { meso- } \\
\text { eutrof }\end{array}$ \\
\hline Nitzschia paleacea GRUNOW & $2,5,9,13$ & 2 & $\begin{array}{l}\text { meso- } \\
\text { eutrof }\end{array}$ \\
\hline Nitzschia perminuta GRUNOW & $5,9,10,11$ & 2 & $\begin{array}{l}\text { meso- } \\
\text { eutrof }\end{array}$ \\
\hline Nitzschia pusila GRUNOW & 14 & 1 & eutrof \\
\hline Nitzschia recta HANTZSCH & 5 & 1 & eutrof \\
\hline Nitzschia subacicularis HuST. & $1,2,6,7,10,12,13,15$ & 3 & $\begin{array}{l}\text { meso- } \\
\text { eutrof }\end{array}$ \\
\hline Nitzschia tubicola GRUNOW & $2,3,4,5,9,11,15$ & 3 & $\begin{array}{l}\text { meso- } \\
\text { eutrof }\end{array}$ \\
\hline Pinnularia braunii (GRUNOW) CLEVE & 1 & 1 & meso \\
\hline Pinnularia divergens W. SмIтн & 6 & 1 & $\begin{array}{l}\text { meso- } \\
\text { eutrof }\end{array}$ \\
\hline Pinnularia gibba EHRENB. & 7,9 & 1 & $\begin{array}{l}\text { meso- } \\
\text { eutrof }\end{array}$ \\
\hline Pinnularia intermedia (LAGERST.) CleVE & $1,8,13$ & 1 & $\begin{array}{l}\text { meso- } \\
\text { eutrof }\end{array}$ \\
\hline Pinnularia interrupta W. SMITH & 7,8 & 1 & eutrof \\
\hline Pinnularia legumen EHRENB. & 8 & 1 & eutrof \\
\hline Pinnularia maior (KÜTZ.) RABENH. & $4,8,9,10$ & 2 & $\begin{array}{l}\text { meso- } \\
\text { eutrof }\end{array}$ \\
\hline $\begin{array}{l}\text { Pinnularia microstauron (EHRENB.) } \\
\text { ClEVE }\end{array}$ & $5,10,14$ & 1 & eutrof \\
\hline Pinnularia subcapitata GREG. & 1 & 1 & meso \\
\hline Pinnularia viridis (NITZSCH) EHRENB. & $1,8,15$ & 1 & $\begin{array}{l}\text { meso- } \\
\text { eutrof }\end{array}$ \\
\hline Pleurosigma salinnarum GRUNOW & 12 & 1 & eutrof \\
\hline $\begin{array}{l}\text { Sellaphora AULDREEKIE MANN \& } \\
\text { MCDONALD }\end{array}$ & $2,5,10,13,14$ & 2 & eutrof \\
\hline Sellaphora ,big”" & 9 & 1 & $\begin{array}{l}\text { meso- } \\
\text { eutrof }\end{array}$ \\
\hline $\begin{array}{l}\text { Sellaphora blackfordensis MANN \& } \\
\text { DROOP }\end{array}$ & 9,10 & 1 & $\begin{array}{l}\text { meso- } \\
\text { eutrof }\end{array}$ \\
\hline Sellaphora „,blunt capitata" & 1 & 1 & meso \\
\hline $\begin{array}{l}\text { Sellaphora capitata MANN \& } \\
\text { MCDONALD }\end{array}$ & 5,9 & 1 & $\begin{array}{l}\text { meso- } \\
\text { eutrof }\end{array}$ \\
\hline Sellaphora „elliptic” & $5,7,13$ & 1 & eutrof \\
\hline Sellaphora pupula (KÜTZ.) MERESCHK. & $2,5,9,14$ & 2 & $\begin{array}{l}\text { meso- } \\
\text { eutrof }\end{array}$ \\
\hline
\end{tabular}




\begin{tabular}{|c|c|c|c|}
\hline Cont. Table 3 & & & \\
\hline Stauroneis acuta W. SMITH & 4 & 1 & meso \\
\hline Stauroneis anceps EHRENB. & 4,11 & 1 & $\begin{array}{l}\text { meso- } \\
\text { eutrof }\end{array}$ \\
\hline $\begin{array}{l}\text { Stauroneis phoeniceteron (NITZSCH) } \\
\text { EHRENB. }\end{array}$ & 11 & 1 & eutrof \\
\hline Stauroneis smithii GRUNOW & 5 & 1 & eutrof \\
\hline Stephanodiscus dubius (FRICKE) HUST. & 10 & 1 & eutrof \\
\hline Stephanodiscus hantzschii GRUNOW & 2 & 1 & eutrof \\
\hline $\begin{array}{l}\text { Stephanodiscus parvus STOERMER \& } \\
\text { HÅK. }\end{array}$ & 3 & 1 & eutrof \\
\hline Surirella angustata KÜTZ. & $1,2,5,7,9,14$ & 2 & $\begin{array}{l}\text { meso- } \\
\text { eutrof }\end{array}$ \\
\hline Surirella bohemica MALY & 1 & 1 & meso \\
\hline $\begin{array}{l}\text { Surirella BREBISSONI KRAMMER \& } \\
\text { LANGE-BERT. }\end{array}$ & $5,7,10$ & 1 & eutrof \\
\hline Surirella lapponica A. CLEVE & 4 & 1 & meso \\
\hline Surirella minuta BRÉB. & 9 & 1 & $\begin{array}{l}\text { meso- } \\
\text { eutrof }\end{array}$ \\
\hline Surirella subsalsa W. SмIтн & 5 & 1 & eutrof \\
\hline Tabellaria fenestrata (LYNGB.) KÜTZ. & 1,11 & 1 & $\begin{array}{l}\text { meso- } \\
\text { eutrof }\end{array}$ \\
\hline Tabellaria flocculosa (RоTн) KüTZ. & $1,5,8,9,10,12$ & 2 & $\begin{array}{l}\text { meso- } \\
\text { eutrof }\end{array}$ \\
\hline $\begin{array}{l}\text { Tetracyclus rupestris (A. BRAUN) } \\
\text { GRUNOW }\end{array}$ & 4 & 1 & meso \\
\hline
\end{tabular}

\section{Acknowledgement}

This research was supported by the GA CR 206/07/0115 project.

\section{References}

Finlay, B.J., Monaghan, E.B. \& Maberly, S.C. (2002): Hypothesis: the rate and scale of dispersal freshwater diatom species is a function of their global abundance. - Protist 153: 261-273.

HINDÁK, F. (ed.) (1978): Sladkovodné riasy. - 714 pp., Státní pedagogické nakladatelství, Bratislava.

Kitner, M. \& PoulíčKovÁ, A. (2000): Řasy několika rybníků v okolí Protivanova [Algae of several fishponds near Protivanov (Moravia, Czech republic)]. - Př́rodovědné studie Muzea Prostějovska 3: 45-53.

KitNer, M. \& PoulíčKovÁ, A. (2003): Littoral diatoms as indicatiors for the eutrophication of shallow lakes. Hydrobiologia 506-509: 519-524.

KoMÁrKOVÁ, J. \& MARVAN, P. (1987): The role of algae in the littoral zone of carp ponds. - Arch. Hydrobiol. Beih. 27: 239-249.

KoŘíneK, V., FotT, B., Fuksa, J., Lellák, J. \& PraŽÁKOvÁ, M. (1987): Carp ponds of Central Europe. - Elsevier Sci. Pub. B. V., Amsterdam: 29-61.

Krammer， K. \& LANGe-Bertalot, H. (1986): Bacillariophyceae. 1. Teil. In Süsswasserflora von Mitteleropa. - In: Ettl, H., Gerloff, J., Heynig, H. \& Mollenhauer, D. (eds), G. Fischer Verlag, Stuttgart, 2/1: 1-876 pp.

Krammer， K. \& LANGe-Bertalot, H. (1988): Bacillariophyceae. 2. Teil. In Süsswasserflora von Mitteleropa. - In: Ettl, H., Gerloff, J., Heynig, H. \&
Mollenhauer, D. (eds), G. Fischer Verlag, Stuttgart, 2/2: 1-596 pp.

Krammer, K. \& Lange-Bertalot, H. (1991a): Bacillariophyceae. 3. Teil. In Süsswasserflora von Mitteleropa. - In: Ettl, H., Gerloff, J., Heynig, H. \& Mollenhauer, D. (eds), G. Fischer Verlag, Stuttgart, 2/3: $1-576 \mathrm{pp}$.

Krammer, K. \& Lange-Bertalot, H. (1991b): Bacillariophyceae. 4. Teil. In Süsswasserflora von Mitteleropa. - In: Ettl, H., Gerloff, J., Heynig, H. \& Mollenhauer, D. (eds), G. Fischer Verlag, Stuttgart, 2/4: 1-437 pp.

ManN, D.G. (1999): The species koncept in diatoms (Phycological Reviews 18). - Phycologia 38: 437495.

PoulíčKovÁ, A., Kitner, M., HaŠler, P., PAKostová, A., KARABINOVÁ, H. \& KŘ́žžovÁ, B. (2003): Fishpond trophic status assessment based on nutriens and bioindication I. Phytoplankton. - Czech Phycology 3: 97-110.

PoulíčKovÁ, A. \& MANN, D.G. (2006): The sources of diatom morphological variability and its importance in biomonitoring. - Book of Abstracts 6th Internat. Symposium Use of Algae for monitoring Rivers., Balatonfured, Hungary, p. 120.

Round, F. E. (1953): An investigation of two benthic algal communities in Malham Tarn, Yorkshire. - J. Ecol. 41: 174-179.

RotT, E. (ed.) (1999): Indikationslisten für Aufwuchsalgen in österreichischen Fliessgewässern. Teil 2: Trophieindikation sowie geochemische Präferenz, taxonomische und toxikologische Anmerkungen, Wien, 247 pp.

(C) Czech Phycological Society (2007)

Received December 10, 2006

Accepted February 27, 2007 
